\title{
Novel image markers for non-small cell lung cancer classification and survival prediction
}

\author{
Hongyuan Wang ${ }^{1}$, Fuyong Xing ${ }^{2,3}$, Hai Su${ }^{4}$, Arnold Stromberg ${ }^{1}$ and Lin Yang ${ }^{2 *}$
}

\begin{abstract}
Background: Non-small cell lung cancer (NSCLC), the most common type of lung cancer, is one of serious diseases causing death for both men and women. Computer-aided diagnosis and survival prediction of NSCLC, is of great importance in providing assistance to diagnosis and personalize therapy planning for lung cancer patients.

Results: In this paper we have proposed an integrated framework for NSCLC computer-aided diagnosis and survival analysis using novel image markers. The entire biomedical imaging informatics framework consists of cell detection, segmentation, classification, discovery of image markers, and survival analysis. A robust seed detection-guided cell segmentation algorithm is proposed to accurately segment each individual cell in digital images. Based on cell segmentation results, a set of extensive cellular morphological features are extracted using efficient feature descriptors. Next, eight different classification techniques that can handle high-dimensional data have been evaluated and then compared for computer-aided diagnosis. The results show that the random forest and adaboost offer the best classification performance for NSCLC. Finally, a Cox proportional hazards model is fitted by component-wise likelihood based boosting. Significant image markers have been discovered using the bootstrap analysis and the survival prediction performance of the model is also evaluated.

Conclusions: The proposed model have been applied to a lung cancer dataset that contains 122 cases with complete clinical information. The classification performance exhibits high correlations between the discovered image markers and the subtypes of NSCLC. The survival analysis demonstrates strong prediction power of the statistical model built from the discovered image markers.
\end{abstract}

Keywords: Lung cancer, Segmentation, Classification, Image informatics, Survival analysis

\section{Background}

Lung cancer is one of the most frequent cancers worldwide. Similar to breast cancer in female, lung cancer is the leading cancer in males, with $17 \%$ of the total new cancer cases and $23 \%$ of the total cancer deaths. The prognosis of lung cancer is still poor, with five-year survival rate of approximately $10 \%$ in most countries. Lung cancer can be classified as small cell lung cancer (SCLC) and non-small cell lung cancer (NSCLC). NSCLC accounts for the majority (84\%) of lung cancer [1]. Two major types of NSCLC are adenocarcinoma (including bronchi alveolar carcinoma) representing about $40 \%$ and squamous cell carcinoma representing about 25-30\% [2]. Accurate

\footnotetext{
${ }^{*}$ Correspondence: linyang711@gmail.com

2 J. Crayton Pruitt Family Department of Biomedical Engineering, University of Florida, 1275 Center Drive, 32611 Gainesville, FL, USA

Full list of author information is available at the end of the article
}

classification and survival analysis can provide assistance for personalized treatment planning and prognosis.

Histopathology images serve as a golden standard for lung cancer diagnosis since they can provide a comprehensive view of the disease and its effect on human tissue [3]. Figure 1 shows some representative images of squamous cell carcinoma and adenocarcinoma. Currently, pathologists make diagnosis decision based on cellular and inter-cellular level morphology. Most of current pathology diagnosis is still based on subjective opinions of pathologists and the varying abilities of doctors could result in large interpretation errors or bias. The proposed framework, which focuses on automated quantitative analysis of histopathology images, could alleviate the subjectivity in NSCLC diagnosis and provides supports to doctors in lung cancer classification and patients' survival analysis. 

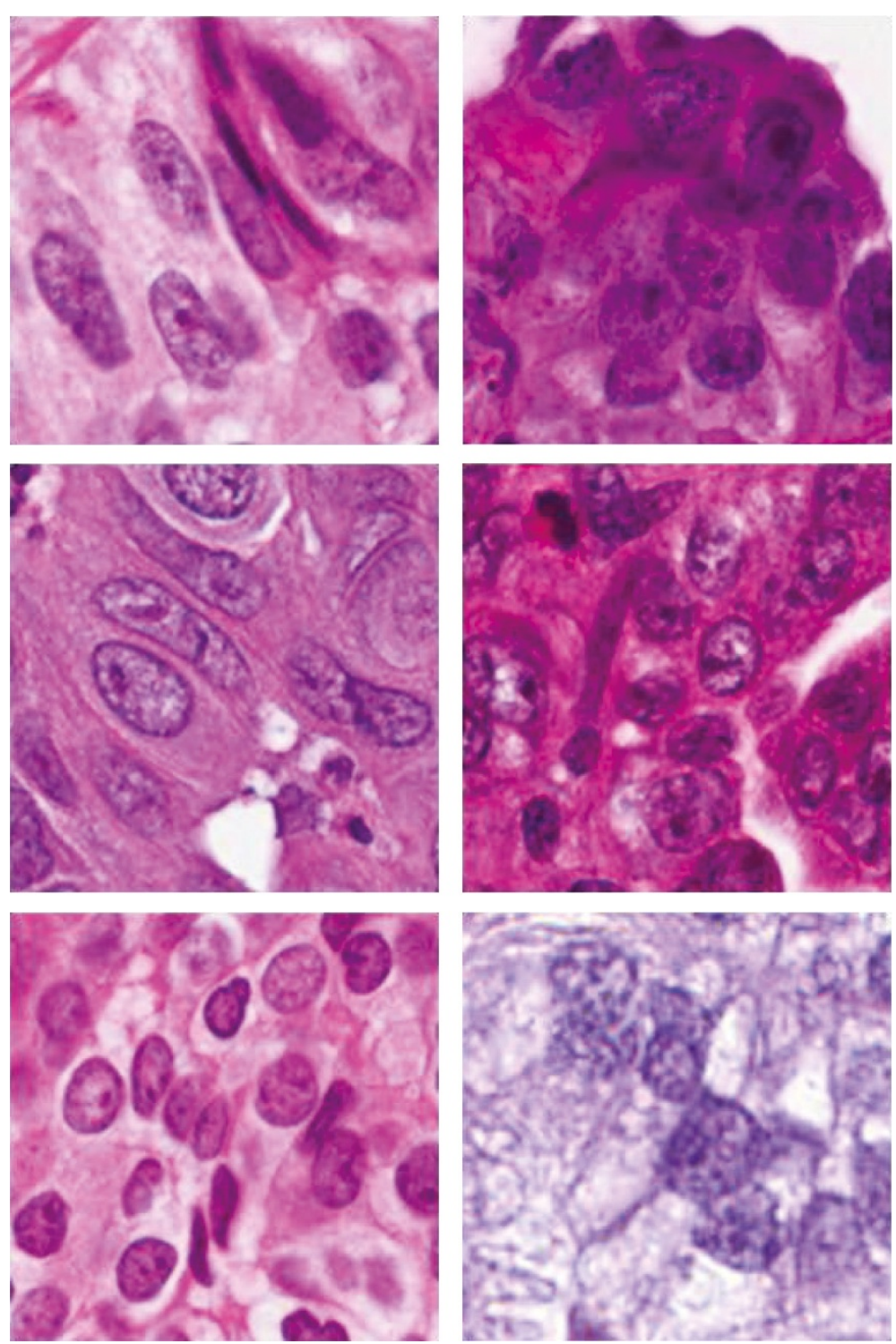

Figure 1 Example Images of squamous cell carcinoma (Left) and adenocarcinoma (Right). Notice: 1. Elongated or spindle cells are more abundant in squamous; 2. Squamous has more clear cell boarders; 3. Squamous usually are more pink while Adeno are more purple or blue.

Recently, there are much active research in imaging informatics [4-12]. Before computer-aided lung cancer diagnosis and survival analysis, usually accurate image segmentation $[13,14]$ is a prerequisite. Sometimes the explicit segmentation may not be required for the applications when the tumor microenvironment is critical for tumor classification; however, in our study, we find that explicit cell localization and cellular features are important for NSCLC classification and survival analysis.

Because crowding and overlapping cancer cells often present significant challenges for most traditional automatic segmentation methods. A vast variety of algorithms based on watershed and its variants [15-17], graph cut
$[18,19]$, and active contour models [20-22] have been proposed. However, none of these methods could robustly handle touching cell segmentation challenges exhibited in lung cancer images. Lu et al. [23] has proposed a supervised learning-based segmentation algorithm to support new image features extraction and polyp detection on CT images, and a flexible, hierarchical feature learning framework integrating different levels of discriminative and descriptive information is presented in [24]. Supervised learning is a potential approach to tackle these challenges, but it requires a lot of labeled training data provided by experienced pathologists. For computer-aided classification, genetic algorithms (GAs) and support vector 
machines (SVMs) have been combined for multi-class cancer identification based on microarray dataset [25]. Partial least square regression (PLSR) and support vector machine with recursive feature elimination (SVM-RFE) have been applied to lung cancer subtype classification [26]. In [27], the lung cancer image classification is modeled as a multi-class multi-instance learning problem, and an adaboost algorithm has been used to perform classification with a bag of feature model. None of these studies correlated image features with the patient survival information.

Survival analysis is related to death in biological organisms and failure in mechanical systems. Several commonly used survival analysis methods are the Kaplan-Meier method for estimating the survival function [28], the logrank test for comparing the equality of two or more survival distributions, and the Cox proportional hazards $(\mathrm{PH})$ model for examining the covariate effects on the hazard function [29]. In survival analysis, one important issue that needs to be considered is censoring problem (subjects are censored if they are not followed up or if the study ends before they die or have an outcome of interest). Cox proportional hazards model [30] is one of the most commonly used multivariate approaches to analyze the survival time data in medical research. It is a semi-parametric method that does not need a specific baseline hazard function and has the capability to effectively handle censoring problem. In other words, it is not necessary to specify a survival distribution to model the effect of the explanatory variables on the duration variable.

In survival analysis, researchers also considers clinical factors or other environmental information [29,31-33]. For example, standard Cox proportional hazards survival model with a spatial random effect extension has been applied and proved that the long-term exposure to combustion-related fine particulate air pollution is an important environmental risk factor for cardiopulmonary and lung cancer mortality [31]. Gene signature expressions have also been used as covariates to conduct survival analysis [34-39] to search for pairs of genes (biomarkers) that are significantly related to patient death.

In this paper, we will present an integrated framework that investigates the prognostic effects of image markers. First, a novel seed detection-based repulsive deformable model is proposed to separate touching cells; secondly, a set of geometry, pixel intensity, and image texture features are extracted to describe cellular morphological properties; thirdly, eight different classification techniques are comparatively analyzed for computer-aided diagnoses of NSCLC; finally, survival analysis is conducted based on a Cox model fitted by component-wise likelihood based boosting. The entire system is designed to assist doctors for more objective and accurate diagnoses and prognoses of NSCLC. Unlike gene sequencing, histopathological slides are always available for each lung cancer patient in routing clinical diagnosis, and therefore the adoption of the developed prediction model does not require any changes to current clinical practice.

The experiments in the paper are conducted using the adenocarcinoma and squamous cell carcinoma lung cancer images downloaded from the TCGA Data Portal. TCGA (The Cancer Genome Atlas) is a collection of cancer specimens, with additional clinical information about participants, metadata about the samples, histopathology slide images from sample portions and molecular information derived from the samples. It is supervised by National Cancer Institute (NCI) and National Human Genome Research Institute (NHGRI) and freely available to researchers.

\section{Methods}

\section{Cell detection and segmentation}

Seed detection is the first critical step for marker-based segmentation methods. Motivated by [22], we have proposed a multi-scale distance map-based voting algorithm for cell detection. The newly developed method can efficiently handle relatively large cell size and shape variation. For each point $(x, y)$, we define a cone-shape voting area $A$ with vertex at $(x, y)$ and votes towards the negative gradient direction of the vertex. A 2D Gaussian kernel $K(m, n, \mu, \Sigma)$ is introduced to weight the voting points:

$$
V(x, y)=C_{1} \sum_{c} \sum_{(m, n) \in S} \mathbf{1}_{A_{c}(m, n)}(x, y) \cdot g D(x, y, \sigma) K(m, n, \mu, \Sigma),
$$

where $C_{1}$ is the normalized constant, $S$ represents the set of all voting points, $A_{c}(m, n)$ denotes the cone-shape voting area with vertex $(m, n)$ and scale $c$. The voting area at each scale is defined as the radial range $\left(r_{\min }, r_{\max }\right)$ and angular range $\Delta, \mu=$ $\left(x+\frac{\left(r_{\max }-r_{\min }\right) \cos \theta}{2}, y-\frac{\left(r_{\max }-r_{\min }\right) \sin \theta}{2}\right)(\theta$ is the angle of the gradient direction with respect to $x$ axis) is the mean of the Gaussian kernel. $\Sigma=\sigma^{2} I_{2}\left(I_{2}\right.$ is the identity matrix) is the covariance matrix. $\mathbf{1}_{A_{c}(m, n)}(x, y)$ is the indicator function: 1 for $(x, y) \in A_{c}(m, n)$ and 0 otherwise. $g D(x, y, \sigma)$ represents the Euclidean distance map. After the confidence map $V(x, y)$ is calculated, we remove those points with relatively lower voting values, which locate near the cell boundaries. In order to achieve a robust seed detection, we apply mean shift [40] to locate the final positions of cell seeds.

Using the boundaries of detected cell seeds as initializations, a novel repulsive balloon snake (RBS) algorithm based on a deformable model [41] is used to seek the cell boundaries. RBS is a parametric model which can naturally preserve cell topologies and prevent contours from splitting or merging with one another. 
A snake is an active curve as $v(s)=(x(s), y(s)), s \in[0,1]$, moving through the image domain to minimize its energy functional, under the influence of internal and external forces. To enforce snakes to inflate or deflate, a pressure force to propose the balloon snake (BS) model was introduced [41]. The external force $F_{\text {ext }}^{B}$ is calculated by:

$$
F_{\text {ext }}^{B}=\gamma \boldsymbol{n}(s)-\lambda \frac{\nabla E_{\text {ext }}(v(s))}{\left\|\nabla E_{\text {ext }}(v(s))\right\|},
$$

where $\boldsymbol{n}(s)$ represents the normal vector (pressure force) to the curve at the specific point on $v(s)$ and $\nabla E_{\text {ext }}(v(s))$ is defined as image force, where $E_{\text {ext }}(v(s))=-\|\nabla I(v(s))\|^{2}$ $(I(v(s))$ is the original image). $\gamma$ and $\lambda$ are the weighting parameters controlling pressure force and image force, respectively.

Balloon snake (BS) model can not be directly used for touching object segmentation. If all balloon snakes move independently, they will cross with one another. Based on these observations, we introduce an interactive scheme to form a RBS model for touching cell segmentation. The intrinsic idea of RBS is based on the following: the cell contour should be driven by its own forces as well as extrinsic forces from other deformable contours; both amplitudes and directions of these extrinsic forces should vary with respect to the distance between snakes. When two snakes are far away, their movements should be dominantly controlled by their own driven forces (internal and external forces); when they get closer, each snake should receive repulsive forces from all other adjacent snakes. As a result, the extrinsic force will prohibit snakes from crossing or merging.

Given an image $I$ with $N$ cells (denoted by $N$ curves $\left.v_{i}, i=1, \ldots, N\right)$, the new repulsive external force $F_{\text {ext }}^{R B}$ for $v_{i}$ is defined as:

$$
\begin{aligned}
F_{\text {ext }}^{R B}= & \gamma \boldsymbol{n}_{\boldsymbol{i}}(s)-\lambda \frac{\nabla E_{\text {ext }}\left(v_{i}(s)\right)}{\left\|\nabla E_{\text {ext }}\left(v_{i}(s)\right)\right\|} \\
& +\omega \sum_{j=1, j \neq i}^{N} \int_{0}^{1} f\left(d_{i j}(s, t)\right) \boldsymbol{n}_{\boldsymbol{j}}(t) d t,
\end{aligned}
$$

where $d_{i j}(s, t)=\left\|v_{i}(s)-v_{j}(t)\right\|_{2}$ is the Euclidean distance between $v_{i}(s)$ and $v_{j}(t) . f(x)>0, x \in(0,+\infty)$, represents a monotonic decreasing function $\left(f(x)=x^{-2}\right.$ in our case), and $\omega$ weights the repulsive force. For a specific point $v_{i}(s)$, the closer it moves to other snakes, the more repulsive forces it will receive. Unlike the original balloon snake, RBS moves contours under the influence of their own driven forces and extrinsic repulsive forces from other snakes. When these two types of forces achieve a balance, snakes stop evolving.

\section{Feature extraction}

Based on the segmented cell boundaries, three groups of cellular features are extracted for subsequent classification and survival analysis. In total we have extracted 166 image morphometric features, which are represented as the candidates of image markers. The detailed notations of feature names and descriptions are listed in the Table 1.

Group 1: Geometry Features. Five geometry features are calculated for each segmented lung cancer cell, including area $A_{\text {cell }}$, contour perimeter $P_{\text {cell }}$, circularity $C=$ $\frac{4 \pi A_{\text {cell }}}{P_{\text {cell }}^{2}}$, major-minor axis ratio, and contour solidity that is defined as the ratio of cell area region over the convex hull defined by the cell boundary.

Group 2: Pixel Intensity Statistics. This group of features are calculated based on the pixels in the segmented cells, including intensity mean, standard deviation, skewness, kurtosis, entropy, and energy. We use Lab color space for better color representation.

Group 3: Texture Features: This group of features contains co-occurrence matrix [42], local binary pattern (LBP) [43], texture feature coding method (TFCM) [44],

\section{Table 1 The image features and their descriptions}

\begin{tabular}{ll}
\hline Name & Description \\
\hline area1-6 & Cell area feature \\
axis1-6 & Major-minor axis ratio \\
cir1-6 & Cell circularity feature \\
peri1-6 & Contour perimeter feature \\
solidity1-6 & Contour solidity feature \\
mean1-6 & Cell intensity mean feature \\
std1-6 & Cell intensity stand deviation feature \\
kurt1-6 & Cell intensity Kurtosis \\
entr1-6 & Cell intensity entropy \\
energy1-6 & Cell intensity energy \\
contrast1-6 & Cell intensity contrast \\
corr1-6 & Cell intensity correlation \\
engy1-6 & Cell intensity energy from co-occurrence matrix \\
homo1-6 & Cell intensity homogeneity \\
skew1-6 & Cell intensity skewness \\
tfcm1-24 & Texture feature coding method (TFCM) \\
csac1-24 & Center symmetric auto correlation (CSAC) feature \\
Ibp1-24 & Local binary pattern (LBP) feature \\
t1-4 & Texton histogram feature \\
\hline
\end{tabular}

The 1-6 in each image feature represent the mean, median, variance and three frequency values of the histogram for each intensity and geometric feature, respectively. Csac, tfcm, lbp and texton histogram features are high dimensional feature vectors, therefore we calculate their moment statistics to reduce the dimensionality. In total we have extracted 166 geometric, pixel intensity, and image texture feature variables for each patient. All variables are normalized before further classification and survival analysis. 
center symmetric auto-correlation (CSAC) [45], and texton features [46]. The co-occurrence matrix [42] is an estimation of the joint probability distribution of intensity of two neighboring pixels. LBP [43] is a measure of local textures. Each pixel in the input image patch is assigned a binary code by comparing the intensity of this pixel to those of its neighbors. Similar to LBP, in TFCM [44], each pixel is assigned a texture feature number (TFN). The TFN of one pixel is generated by comparing this pixel with its neighbors in four directions: $0^{\circ}, 45^{\circ}, 90^{\circ}$, and $135^{\circ}$. A histogram is calculated based on the TFNs of one image patch. CSAC is a measure of the local patterns with symmetrical forms. We calculated a series of local auto-correlation and covariance introduced in [45], including symmetric texture covariance (SCOV) and variance (SVR), and within-pair variance (WVAR) and between-pari variance (BVAR). $3 \times 3$ pixel unit of each channel is considered for CSAC feature. Texton [46] is a discriminative texture representation. The calculation of texton feature is based on unsupervised learning. We randomly picked some cells in each image as training samples. These cell patches are filtered by texton filter bank. $\mathrm{K}$-means clustering is then applied and the centers of the clusters are defined as textons. To generate the texton histogram for a new image, the image is first filtered by the same texton filter bank, then each pixel is assigned to one texton to build the final texton histogram.

\section{NSCLC classification}

After calculating the aforementioned image features, we first perform the NSCLC subtype classification. In this stage, several conventional machine learning methods and recently published state-of-the-art algorithms that can handle high dimensional data are compared, which include multiple support vector machine recursive feature extraction (MSVM-RFE) algorithm [47], L1 penalized logistic regression [48,49], random forest [50,51], naive Bayesian [52,53], adaboost [54,55], sparse coding spatial pyramid matching (ScSPM) alogrithm [56], localityconstrained linear coding (LLC) [57], and nearest class mean (NCM) classifier [58]. MSVM-RFE is an iterative feature selection method that uses a backward elimination procedure. Resampling scheme is introduced to stabilize the feature rankings. At each iteration, the feature ranking score is computed based on the weight vectors of multiple linear SVMs trained on subsamples of the original training data and the feature with the smallest ranking score is removed from the model. L1 penalized logistic regression provides an efficient lasso regularization path for logistic regression, which enables feature shrinkage and selection for high dimensional data. Random forest is an ensemble learning method for classification, which can generate a score by permutation to rank the importance of variables in classification problem. Naive
Bayesian classifier is a simple probabilistic classifier based on the Bayes theorem with naive feature conditional independence assumptions. The adaboost algorithm employs the idea of sequentially applying a classification algorithm to reweighted versions of the training data and then taking a weighted majority vote of a ensemble of weak classifiers. Adaboost can provide an importance score for each weak classifier that corresponds to one selected feature. ScSPM is an extension of spatial pyramid matching [59] and the algorithm uses selective sparse coding followed by multi-scale spatial max pooling and SVM. LLC is another feature representation method that applies locality constraint to project each feature into a sparse code. NCM is a distance-based classification with projecting the features into a low-dimensional space for classification. These three recent algorithms have already made remarkable successes on a range of nature image classification benchmarks.

\section{Survival analysis}

Before survival analysis, dimension reduction is a widely used approach to avoid the "curse of dimensionality". Common examples of linear dimension reduction methods, such as principal component analysis (PCA), are proposed to minimize the variances. Meanwhile, least absolute shrinkage and selection operator (LASSO) [60] method is another classic method of feature shrinkage and selection for regression that can potentially handle high dimensional data. Least angle regression (LARS) is proposed for variable selection in the linear regression setting for high dimensional data [61]. The LARS selects predictors by its current correlation or angle with the response, where the correlation is defined as the co-correlation between the predictor and the current residuals. Moreover, elastic net is proposed as a new regularization and variable selection method for feature selection [62]. Boosting is another widely used feature selection approach. It applies the idea of fitting an ensemble of weak learners to the data. Furthermore, component-wise boosting has been proposed to estimate the model with intrinsic variable selection [63]. The term component-wise means each base learner only consists of linear function of one component (variable). For each covariate, a base-learner is specified and only the best base-learner is updated in each boosting step. Finally only part of base learners are chosen to ensemble the strong classifier when the optimal boosting iteration is reached. The algorithm can generate a strong classifier and a sparse set of selected features.

Given the observations $\left(t_{i}, d_{i}, x_{i}\right), i=1,2, \ldots, n$, where $t_{i}$ is the observed time to the event of interest for individual $i, d_{i}$ equals 1 if an event occurred at that time and 0 if the observation has been censored, and $x_{i}$ is vector of covariates obtained at time 0 . The component-wise likelihood 


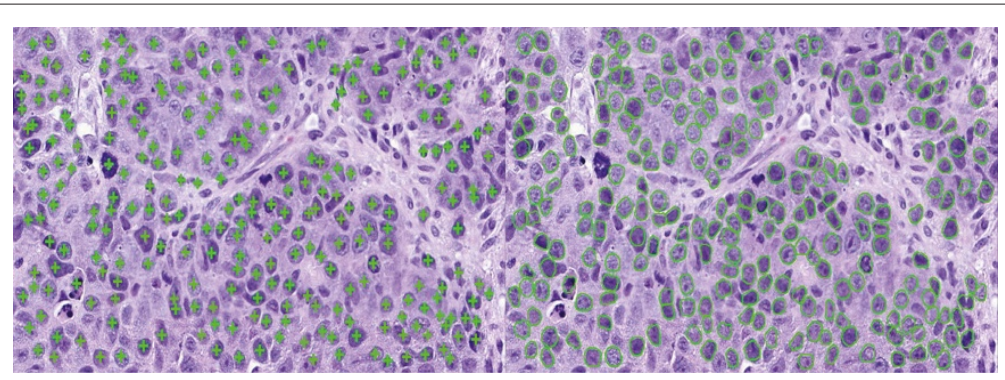

Figure 2 Cell detection (left) and segmentation (right) results. Please note that the cells with small areas correspond to non-tumor cells, and are automatically removed after the boundaries are extracted. Those false detected seeds locating in the lymphocyte regions are also removed using a simple intensity threshold before cell segmentation is conducted.

based boosting algorithm for high dimensional survival analysis is based on the Cox proportional hazards model:

$$
\lambda(t)=\lambda_{0}(t) e^{\beta^{\prime} x},
$$

where $\lambda_{0}(t)$ is the baseline hazard and $x$ is covariate vector. For estimation, the baseline hazard $\lambda_{0}(t)$ is left unspecified and the estimate of $\beta$ is obtained by maximizing the partial log likelihood.

$$
l(\beta)=\sum_{i=1}^{n} d_{i}\left(\beta^{\prime} x_{i}-\log \left(\sum_{j=1}^{n} I\left(t_{j} \leq t_{i}\right) \exp \left(\beta^{\prime} x_{i}\right)\right)\right),
$$

For high dimensional data, penalized regression methods like LASSO [60], ridge regression [64], would add a penalization term into the partial log likelihood function and the penalized partial likelihood is maximized by techniques such as quadratic programming.

In this paper, we apply component-wise likelihood based boosting [65] to dimensionality reduction, which adapts from the offset-based boosting approach from [66]. In each iteration the previous boosting steps are incorporated as an offset in a penalized partial likelihood estimation. Component-wise indicates that only one single parameter, i.e., one covariate, is updated in every iteration by maximization the L2 penalized partial log likelihood with respect to each candidate covariate.

$$
l_{p e n}(\beta)=l(\beta)-\lambda \beta^{\prime} I_{p} \beta,
$$

where $I_{p}$ is a diagonal matrix to penalize each covariate separately, with diagonal elements equal to 1 for each candidate and 0 for the rest corresponding to penalization and no-penalization. The candidate covariate that can best improve the overall fitting will be selected for updating. As the number of boosting steps increases, more feature variables will be selected and chosen with respect to their relevances in predicting survival rates. The result is expected to be sparse with many coefficients equal to zero. The coefficient paths of component-wise boosting are expected to be more stable than LASSO based approaches [65]. In addition, it has two major advantages over LASSO: 1) it allows for unpenalized mandatory covariates; 2) it can handle correlated covariates by including pathway information [67].

\section{Results}

\section{Cell detection and segmentation}

The cell detection and segmentation results are displayed in Figure 2. It can be observed that even for heavily touched regions, cells are still accurately detected and segmented automatically. It is worth mentioning that the proposed cell detection algorithm can handle relatively large size variations, and the repulsive snake models can prevent contours from overlapping with one another.

We have compared the proposed voting method (SPV) presented in [22] and the phase-coded hough transform (HT) based on quantitative measurement. In our evaluation a positive detection is counted if a detected seed locates within a 8-pixel circle around a ground truth seed; otherwise, a miss is counted. To measure the accuracy of the cell detection algorithms, we compute the mean, variance, maximum and minimum of the distance between the detected seeds and their corresponding ground truth seeds. In addition, we also show miss rate (MR) and false positive rate (FP) in Table 2. The ground truth seeds are manually generated for comparison. As one can see, the improved voting approach produces the best performance in comparison with other two methods.

Table 2 The pixel-wise seed detection accuracy compared with ground truth

\begin{tabular}{lllllll}
\hline & Mean & Variance & Max & Min & MR & FP \\
\hline HT & 3.9 & 4.13 & 8.0 & 0.19 & 0.46 & 0 \\
\hline SPV & 3.0 & 3.13 & 7.9 & 0.29 & 0.21 & 0.002 \\
\hline Proposed & $\mathbf{2 . 6}$ & $\mathbf{2 . 8}$ & 7.9 & $\mathbf{0 . 1 2}$ & $\mathbf{0 . 1 2}$ & 0.002
\end{tabular}

The best performance measured in each metric is marked in boldface. 
Table 3 The performance of segmentation measured by precision and recall

\begin{tabular}{llclll}
\hline $\begin{array}{l}\text { Precision } \\
\text { mean }\end{array}$ & $\begin{array}{l}\text { Precision } \\
\text { variance }\end{array}$ & $\mathbf{8 0 \%}$ & $\begin{array}{l}\text { Recall } \\
\text { mean }\end{array}$ & $\begin{array}{l}\text { Recall } \\
\text { variance }\end{array}$ & $\mathbf{8 0 \%}$ \\
\hline 0.87 & 0.01 & 0.95 & 0.95 & 0.01 & 0.96 \\
\hline
\end{tabular}

To evaluate the performance of the segmentation algorithm, we define precision $P=\frac{s e g \bigcap g t}{s r}$ and recall $R=$ $\frac{s e g \cap g t}{g t}$, where seg represents the segmentation result and $g t$ represents the manually-generated ground truth. We show the mean, variance and $80 \%$ in Table 3 . The segmentation algorithm can effectively handle touching cells and provide accurate segmentation results with high precision and recall rates.

\section{NSCLC classification}

Precison, recall, and accuracy have been used as prediction performance metrics for NSCLC classification. The training (62) and testing (60) datasets have been randomly selected and repeated five times to test the accuracies of classification using the TCGA dataset consisting of 122 NSCLC patients. Table 4 shows the average recall, precision, and accuracy using eight classifiers. The experiments indicate that the random forest and adaboost provide the best results.

To assess the relative importance of the 166 image markers, we have applied adaboost to the entire dataset and generated the frequency score for the variable selected in each boosting iteration. The results are shown in Figure 3. Higher importance score indicates a more representative feature variable for NSCLC classification.

The top 10 features selected by adaboost are $4 \mathrm{lbp}$ features, 3 solidity features, and area3, kurt3 and peri3 features. Among the top 30 features, lbp, area, solidity, axis, tfcm, energy, correlation and contrast are most commonly selected image features. Peri, kurt, std and circularity all have one feature been selected. The ranking suggests that the image texture features and geometric features are representative markers to distinguish between two subtypes of NSCLC: Adenocarcinoma (AC) and Squamous cell carcinoma (SCC). The results also indicate that 1) there are more elongated cells for SCC than AC; 2) AC usually has a relatively larger intensity variation inside cells than SCC; 3) SCC cells are often over-stained and exhibit more clear boundaries; 4) AC cells usually exhibit more inhomogeneous texture than SCC.

\section{Survival analysis}

The TCGA NSCLC dataset contains complete patients' histopathological image information. It has been randomly divided into training $(n=65)$ and testing set $(n=57)$. The training set is used to build a Cox regression model with component-wise likelihood based boosting for feature selection. Among 166 image features, we first conduct univariate Cox regression and abandon those with Wald test $p$ value less than a threshold (0.25). The rest image features are chosen as candidate markers to conduct component-wise likelihood based boosting for Cox Proportional Hazards Model. After the univariate Cox regression step, 59 image features have been selected as candidates. The penalty parameter $\lambda$, which determines the size of the boosting steps, is determined based on cross validation. Six-fold cross validation on the training set has been performed to choose the number of boosting steps $M$ (Figure 4). The final representative image markers selected are energy $5, \mathrm{lbp} 5, \mathrm{lbp} 24$, homo3, homo5, tfcm 4 and skew6 with corresponding coefficients $-0.0268,0.1670,0.0343,0.0685,-0.1382$, 0.1130 and -0.2150 . Please note that all these feature covariates selected belong to pixel intensity features and texture features. This demonstrates that cell staining and inhomogeneity inside the nuclei, which may indicate the protein structures of the cancer cells, hold strong potential to predict NSCLC patients' survival.

After the prediction model training procedure, we have employed the time dependent ROC curves for uncensored data and AUC as criteria to select the best thresholds for risk scores and assess how well the model predicts patients' survival outcome. At time $t$, larger AUC indicates better predictability of time to event measured by sensitivity and specificity. After classifying patients into lowand high-risk groups, we can estimate and compare their Kaplan-Meier survival curves. The performance of such a binary classifier is generally evaluated in terms of the overall predictive accuracy.

With the approach mentioned above, the seven-feature prediction model and a binary classifier have been applied to distinguish between the low- and high-risk groups for

Table 4 The average recall, precision and accuracy of NSCLC classification

\begin{tabular}{lllllllll}
\hline & MR & PL & NB & RF & AB & LLC & SC & NCM \\
\hline Recall (\%) & 89.7 & 75.9 & 71.0 & 93.1 & 92.4 & 75.3 & 66.1 & 74.1 \\
Precision (\%) & 80.5 & 55.9 & 67.3 & 90.6 & 90.9 & 80.4 & 83.2 & 75.4 \\
Accuracy (\%) & 84.3 & 53.6 & 66.4 & 92.0 & 91.7 & 76.7 & 69.7 \\
\hline
\end{tabular}

MR: MSVM-RFE, PL: L1 penalized logistic regression, NB: naive Bayesian, RF: random forest, AB: adaboost, LLC: locality-constrained linear coding, SC: sparse coding spatial pyramid matching, NCM: nearest class mean classifier. 


\section{Variable Importance Plot}

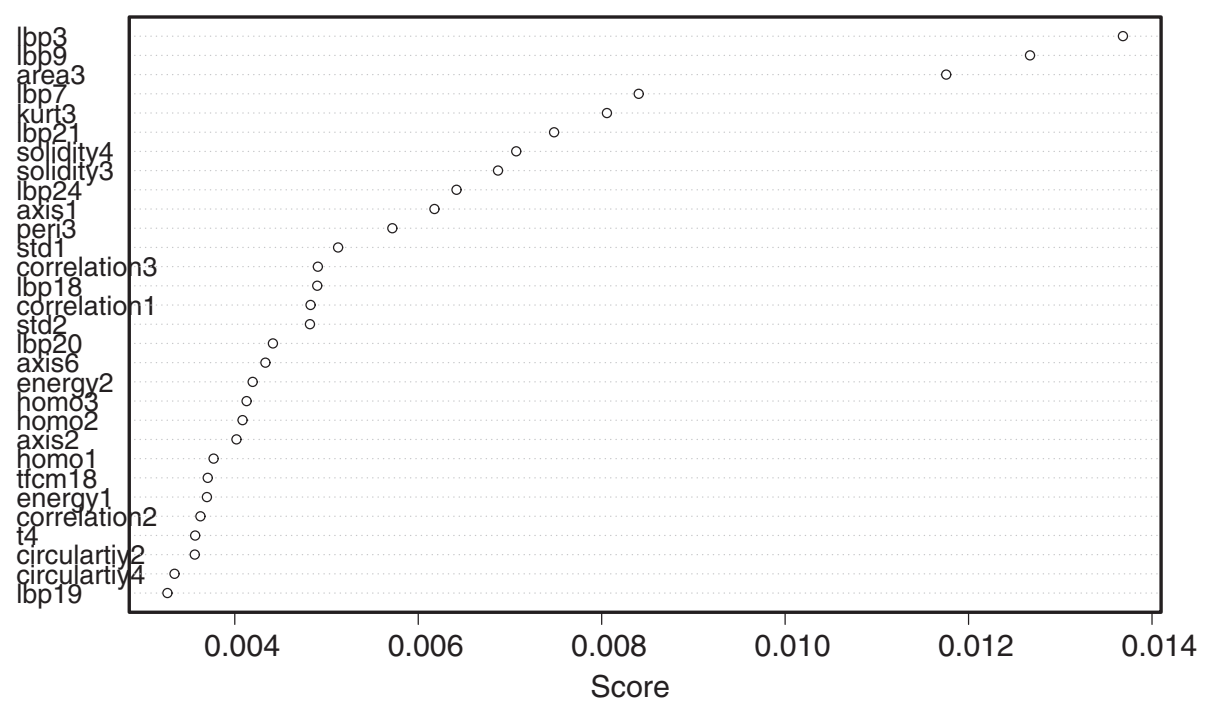

Figure $\mathbf{3}$ The feature importance of the $\mathbf{1 6 6}$ image morphometric features. Only the top 30 image features are shown here. The $x$-axis represents the frequency score and $y$-axis denotes the name of each feature variable.

both training set $(n=65)$ and testing set $(n=57)$. Kaplan-Meier survival curves have been estimated and plotted in Figure 5. The log rank test shows significant difference between two groups. The $p$ value on testing set is slightly larger than the training set. The good performance demonstrates the accurate survival prediction power using this set of discovered image markers.

Using a multivariate Cox proportional hazards model, we have assessed the image markers related risk score in the context of other measured prognostic factors, including age, gender, cancer type, smoking history, and tumor stage. The results are presented in Table 5 and Table 6 . The $p$ value of Wald test of each covariate coefficient suggests that NSCLC subtype and tumor stage are significantly related to survival rate in the multivariate Cox regression without the image marker related risk score. However, when the image marker related risk score is introduced, it becomes the most significant variable in the model. To further quantify how much improvement is gained in survival analysis after the risk score is added, Akaike Information Criterion (AIC) and Bayesian Information Criterion (BIC) of these two models are computed. The experiments show that $\mathrm{AIC}=110.20, \mathrm{BIC}=115.54$ for the first model compared to $\mathrm{AIC}=99.81, \mathrm{BIC}=106.04$ for the model including the risk score. The clear difference demonstrates strong evidence in favor of the prognostic model with image marker related risk score. Hazard ratio is also measured and reported in Table 5 and Table 6 . A
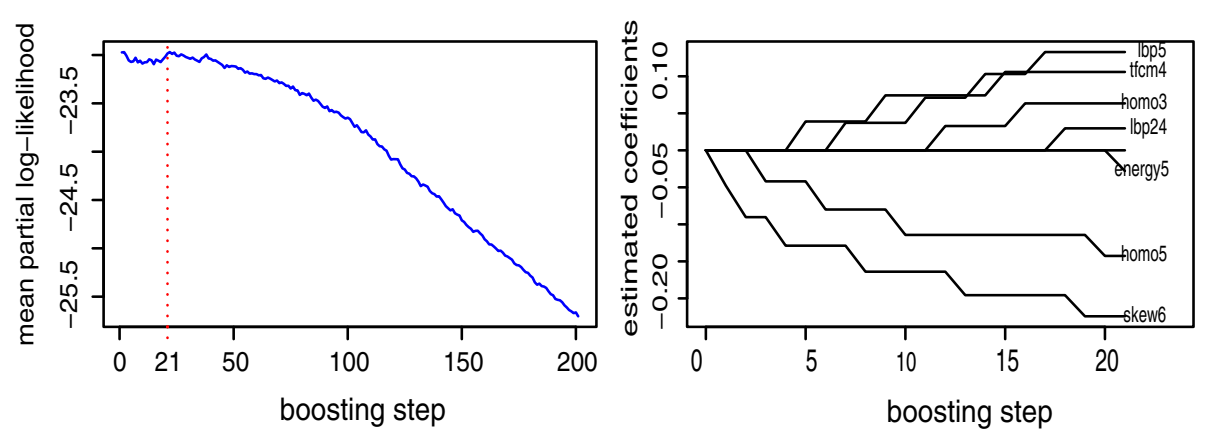

Figure 4 (Left) The plot of mean partial log likelihood against the number of boosting steps using six-fold cross validation and (Right) the plot of coefficient paths, i.e. the parameter estimation plotted against the boosting steps until the optimal steps were reached. $M=21$ is used since it minimizes the mean partial log likelihood. Since we use an offset-based update mechanism, the same image feature might be reselected and its coefficient would be updated. We use branch-out for illustration purpose when one specific image feature is selected. 

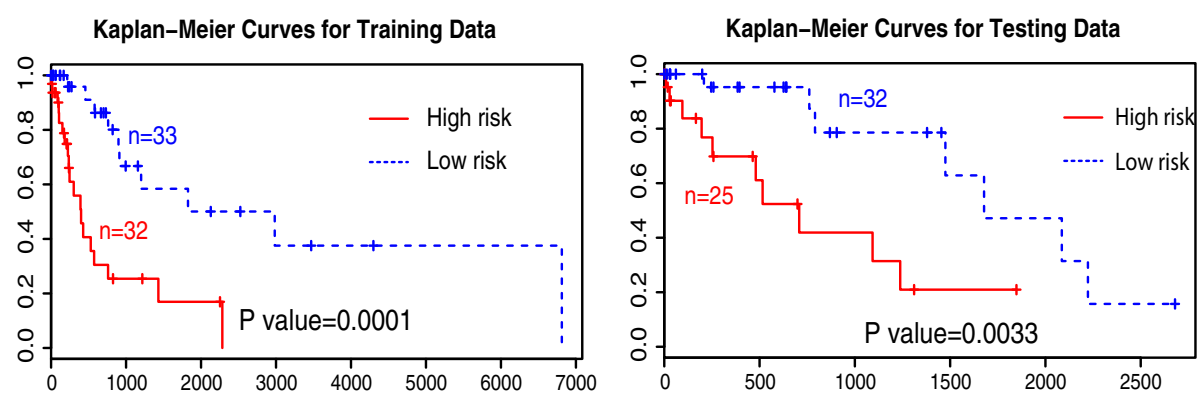

Figure 5 Kaplan-Meier survival curves of two groups classified by predicted risk scores for training (Left) and testing dataset (Right). The $p$ value of log rank test for training is 0.0001 , and for testing is 0.0033 . The $x$ axis is the time in days and the $y$ axis denotes the probability of overall survival.

hazard ratio greater than one, or equivalently, a value of coefficient greater than zero, indicates that as the value of this covariate increases, the event hazard increases and thus the length of survival decreases. Given the proposed comprehensive prediction model, for each NSCLC cancer patient with $H \& E$ stained diagnostic pathology image and clinical information, we can offer a personalized survival function and automatically group the individual into lowor high-risk group with an estimated risk score.

To measure the robustness of the feature selection, we have conducted the bootstrap analysis. We have resampled the whole dataset 5000 times with replacement, performed the boosting feature selection procedure on each sample and counted the frequency of selecting one specific feature variable. The top 10 most frequently selected image markers are: peri6, homo3, homo4, homo5, skew6, lbp5, lbp16, csac6, csac15 and tfcm18. Among the top 10 image features that are most highly associated with survival, 4 are pixel intensity features, 5 are image texture features and only 1 belongs to geometric feature. Moreover, 4 out of the 7 significant features previously selected in the training set are from the top 11 features in bootstrap analysis on the whole set, which shows good consistence of the proposed algorithm.

Univariate survival analysis has been conducted to validate the feature variable selection procedure by showing

Table 5 (TCGA NSCLC testing data $n=57$ ) Multivariate Cox proportional hazards analysis of all clinical covariates without the image feature related risk score

\begin{tabular}{llll}
\hline Variable & p-value & Hazard Ratio & SE (coef) \\
\hline Age & 0.0900 & 0.94 & 0.035 \\
NSCLC subtype & 0.0091 & 0.13 & 0.780 \\
Smoking history & 0.3914 & 0.80 & 0.479 \\
Gender & 0.7928 & 0.45 & 0.733 \\
Tumor stage II & 0.6800 & 1.46 & 0.924 \\
Tumor stage III \& IV & 0.0076 & 0.05 & 1.138 \\
\hline
\end{tabular}

that the selected features are closely related to lung cancer patients' survival time. We choose the median of each image marker as the threshold to group the patients and plot the Kaplan-Meier curves for those two groups. Log rank test is conducted to test the difference of the two curves. It is shown that 4 out of the top 8 image markers selected from the bootstrap analysis can achieve significant $\log$ rank test outcome at $\alpha=0.10$ level while the others is still acceptable even though they do not reach statistical significance for this naive approach (Figure 6). In addition, no single image marker can achieve the same prediction power as the combined risk score using the set of discovered image markers.

\section{Discussion and conclusions}

In this paper, we have investigated novel image markers for both computer aided diagnosis and prognosis of non-small cell lung cancer. We propose an integrated framework that consists of cell detection, segmentation, feature extraction, classification, discovery of image markers, and survival analysis for NSCLC. A multi-scale distance map-based voting algorithm is first introduced to

Table 6 (TCGA NSCLC testing data $n=57$ ) Multivariate cox proportional analysis of all clinical covariates and image feature related risk score

\begin{tabular}{llll}
\hline Variable & p-value & Hazard Ratio & SE (coef) \\
\hline Risk score & 0.0049 & 4.99 & 0.571 \\
Age & 0.1600 & 0.94 & 0.037 \\
NSCLC subtype & 0.0076 & 0.11 & 0.832 \\
Smoking history & 0.6530 & 0.72 & 0.741 \\
Gender & 0.4000 & 0.53 & 0.739 \\
Tumor stage II & 0.6000 & 1.64 & 0.930 \\
Tumor stage III \& IV & 0.0090 & 0.04 & 1.160 \\
\hline
\end{tabular}

Smoking history is a continuous variable representing years of smoking history. Gender is a binary variable ( 0 for male and 1 for female). Cancer type is also a binary variable ( 0 for squamous cell carcinoma and 1 for adenocarcinoma). Tumor stage is a three level categorical variable (stage $I$ is treated as the reference group). 


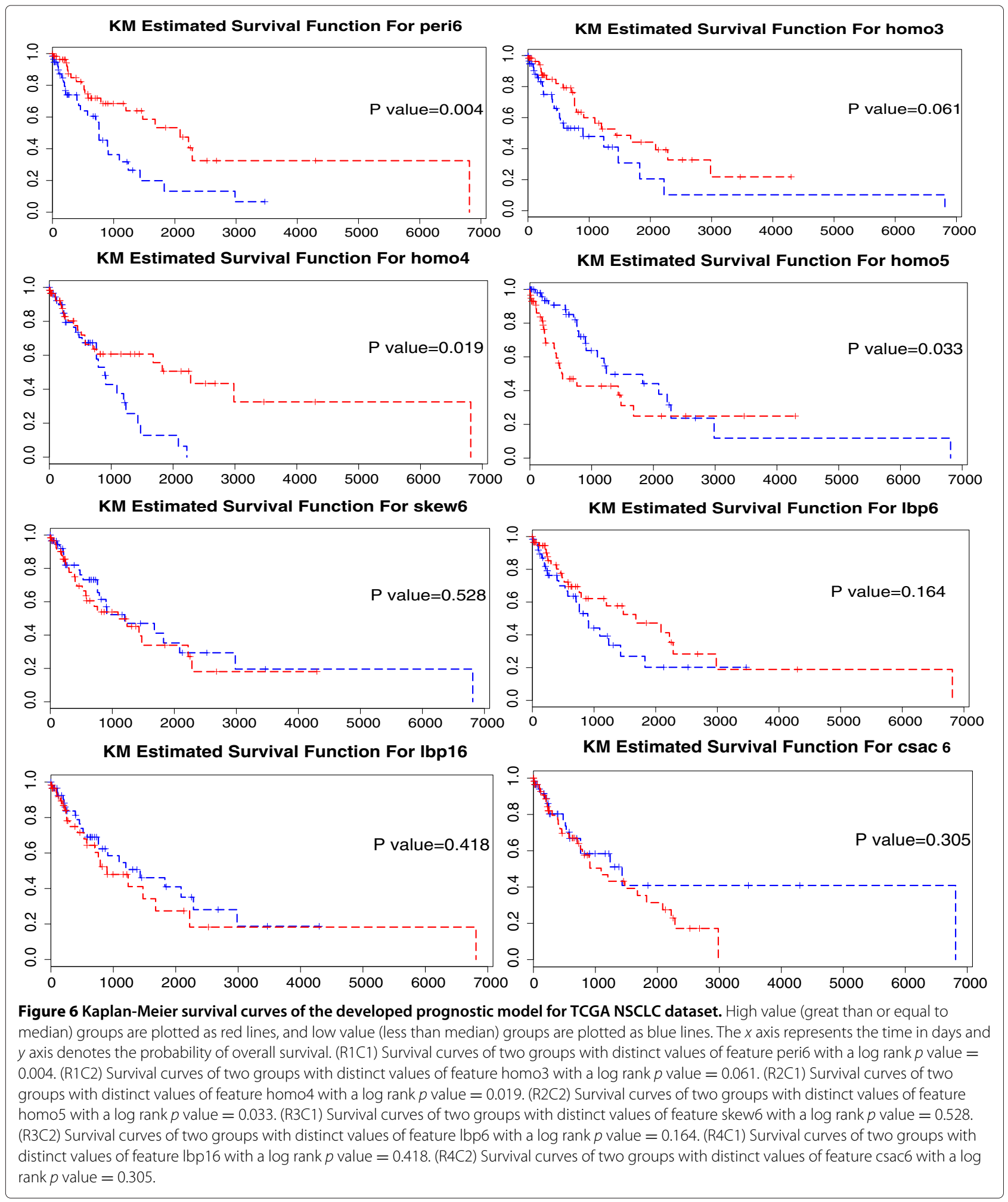

localize individual cells, and a repulsive deformable model is proposed to segment the cells using the previous detection results as initializations. A complete set of cellular features are extracted, and several advanced classification techniques are compared using the image markers calculated in previous steps. Finally, a Cox model fitted with component-wise likelihood based boosting is applied and several survival analysis approaches have been conducted 
to evaluate the discovered image features. Through extensive experiments, we have found a set of diagnostic image markers that are highly correlated to NSCLC subtype classification. In addition, we have also discovered a set of prognostic image markers (majorly representing image staining characteristics and inhomogeneity inside the nuclei of cancer cells) to predict NSCLC patients' survival. We statistically prove that the developed comprehensive image marker related risk score is a strong predictor for patients' survival than traditional clinical factors. Together with clinical information, it provides significant clinical values for patients' prognosis.

\section{Competing interests}

The authors declare that they have no competing interests.

\section{Authors' contributions}

HW conducted the classification and survival analysis experiment. FX and HS conducted the image collection, segmentation and feature extraction experiment. LY participated in the design of study and and coordination and helped to draft the manuscript. AS participated in the design of the study and the statistical analysis section. All authors read and approved the final manuscript.

\section{Acknowledgements}

This research is funded by an Institutional Development Award (IDeA) from the National Institute of General Medical Sciences of the National Institutes of Health under grant number 2 P20 GM103436-14. The project is also partially supported by the National Center for Research Resources and the National Center for Advancing Translational Sciences, National Institutes of Health, through Grant UL1TR000117 (or TL1 TR000115 or KL2 TR000116). The content is solely the responsibility of the authors and does not necessarily represent the official views of the $\mathrm{NIH}$.

\section{Author details}

${ }^{1}$ Department of Statistics, University of Kentucky, 725 Rose Street, 40536 Lexington, KY, USA. ${ }^{2}$ J. Crayton Pruitt Family Department of Biomedical Engineering, University of Florida, 1275 Center Drive, 32611 Gainesville, FL, USA. ${ }^{3}$ Department of Electrical and Computer Engineering, University of Florida, 233 Larsen Hall, 32611 Gainesville, FL, USA. ${ }^{4}$ Department of Computer Science, University of Kentucky, 329 Rose Street, 40536 Lexington, KY, USA.

Received: 3 February 2014 Accepted: 14 August 2014

Published: 19 September 2014

\section{References}

1. Detterbeck FC, Boffa DJ, Tanoue LT: The new lung cancer staging system. CHEST J 2009, 136(1):260-271.

2. Anagnostou VK, Dimou AT, Botsis T, Killiam EJ, Gustavson MD, Homer RJ, Boffa D, Zolota V, Dougenis D, Tanoue L, Gettinger SN, Detterbeck FC, Syrigos KN, Bepler G, Rimm DL: Molecular classification of nonsmall cell lung cancer using a 4-protein quantitative assay. Cancer 2012, 118(6):1607-1618.

3. Gurcan MN, Boucheron LE, Can A, Madabhushi A, Rajpoot NM, Yener B: Histopathological image analysis: a review. IEEE Rev Biomed Eng 2009, 2:147-171.

4. Caicedo JC, González FA, Romero E: Content-based histopathology image retrieval using a kernel-based semantic annotation framework. J Biomed Inform 2011, 44(4):519-528.

5. Díaz G, González FA, Romero E: A semi-automatic method for quantification and classification of erythrocytes infected with malaria parasites in microscopic images. J Biomed Informat 2009, 42(2):296-307.

6. Mazurowski MA, Lo JY, Harrawood BP, Tourassi GD: Mutual information-based template matching scheme for detection of breast masses: from mammography to digital breast tomosynthesis. J Biomed Inform 2011, 44(5):815-823.
7. Wei $\mathrm{C}-\mathrm{H}, \mathrm{Li}$ Y, Huang PJ: Mammogram retrieval through machine learning within bi-rads standards. J Biomed Inform 2011, 44(4):607-614

8. Kim D, Ramesh BP, Yu H: Automatic figure classification in bioscience literature. J Biomed Inform 2011, 44(5):848-858.

9. Wang X, Zheng B, Li S, Mulvihill JJ, Wood MC, Liu H: Automated classification of metaphase chromosomes: optimization of an adaptive computerized scheme. J Biomed Inform 2009, 42(1):22-31.

10. Wang J, Zhou X, Li F, Bradley PL, Chang S-F, Perrimon N, Wong ST: An image score inference system for rnai genome-wide screening based on fuzzy mixture regression modeling. J Biomed Inform 2009, 42(1):32-40.

11. Kothari S, Phan JH, Stokes TH, Wang MD: Pathology imaging informatics for quantitative analysis of whole-slide images. J Am Med Inform Assoc 2013, 20:1099-1108.

12. Peng $H$, Roysam $B$, Ascoli $G$ : Automated image computing reshapes computational neuroscience. BMC Bioinformatics 2013, 14:293.

13. Song Y, Cai W, Huang H, Wang Y, Feng D, Chen M: Region-based progressive localization of cell nuclei in microscopic images with data adaptive modeling. BMC Bioinformatics 2013, 14:173.

14. Zhang W, Feng D, Li R, Chernikov A, Chrisochoides N, Osgood C, Konikoff C, Newfeld S, Kumar S, Ji S: A mesh generation and machine learning framework for drosophila gene expression pattern image analysis. BMC Bioinformatics 2013, 14:372.

15. Zhou X, Liu K-Y, Bradley P, Perrimon N, Wong ST: Towards automated cellular image segmentation for rnai genome-wide screening. In Medical Image Computing and Computer-Assisted Intervention-MICCAI 2005, vol. 3749. Springer Berlin Heidelberg; 2005:885-892.

16. Cheng J, Rajapakse JC: Segmentation of clustered nuclei with shape markers and marking function. IEEE Trans Biomed Eng 2009, 56(3):741-748.

17. Yang $X, L i H$, Zhou $X$ : Nuclei segmentation using marker-controlled watershed, tracking using mean-shift, and kalman filter in time-lapse microscopy. IEEE Trans Circ Syst 2006, 53(11):2405-2414.

18. Bernardis E, Yu S: Finding dots: Segmentation as popping out regions from boundaries. In Computer Vision and Pattern Recognition (CVPR), 2010 IEEE Conference On. San Francisco, CA: IEEE; 2010:199-206.

19. Al-Kofahi Y, Lassoued W, Lee W, Roysam B: Improved automatic detection and segmentation of cell nuclei in histopathology images. IEEE Trans Biomed Eng 2010, 57(4):841-852.

20. Lankton S, Tannenbaum A: Localizing region-based active contours. IEEE Trans Image Process 2008, 17(11):2029-2039.

21. Bergeest J-P, Rohr K: Fast globally optimal segmentation of cells in fluorescence microscopy images. In Medical Image Computing and Computer-Assisted Intervention-MICCAl 2011, vol. 6891. Springer Berlin Heidelberg; 2011:645-652

22. Qi X, Xing F, Foran DJ, Yang L: Robust segmentation of overlapping cells in histopathology specimens using parallel seed detection and repulsive level set. IEEE Trans Biomed Eng 2012, 59(3):754-765.

23. Lu L, Bi J, Wolf M, Salganicoff M: Effective 3D object detection and regression using probabilistic segmentation features in $C T$ images. In Computer Vision and Pattern Recognition (CVPR), IEEE Conference On. Providence, RI: IEEE; 2011:1049-1056.

24. Lu L, Devarakota P, Vikal S, Wu D, Zheng Y, Wolf M: Computer aided diagnosis using multilevel image features on large-scale evaluation. In Medical Computer Vision. Large Data in Medical Imaging. Springer International Publishing Switzerland; 2014:161-174.

25. Peng S, Xu Q, Ling XB, Peng X, Du W, Chen L: Molecular classification of cancer types from microarray data using the combination of genetic algorithms and support vector machines. FEBS Lett 2003, 555(2):358-362.

26. Gao L, Li F, Thrall MJ, Yang Y, Xing J, Hammoudi AA, Zhao H, Massoud Y, Cagle PT, Fan Y, Wong KK, Wang Z, Wong ST: On-the-spot lung cancer differential diagnosis by label-free, molecular vibrational imaging and knowledge-based classification. J Biomed Opt 2011, 16(9):096004-096004

27. Zhu $L$, Zhao B, Gao Y: Multi-class multi-instance learning for lung cancer image classification based on bag feature selection. In Fuzzy Systems and Knowledge Discovery (FSKD), 2008 IEEE Fifth International Conference On. Volume 2; 2008:487-492.

28. Kaplan EL, Meier P: Nonparametric estimation from incomplete observations. J Am Stat Assoc 1958, 53(282):457-481. 
29. Fleming TR, Lin D: Survival analysis in clinical trials: past developments and future directions. Biometrics 2000, 56(4):971-983.

30. Cox DR: Regression models and life-tables. J Roy Stat Soc B 1972, 34:187-220.

31. Pope CA, Burnett RT, Thun MJ, Calle EE, Krewski D, Ito K, Thurston GD: Lung cancer, cardiopulmonary mortality, and long-term exposure to fine particulate air pollution.. JAMA 2002, 287(9):1132-1141.

32. Dockery DW, Pope CA, Xu X, Spengler JD, Ware JH, Fay ME, Ferris BG, Speizer FE: An association between air pollution and mortality in six us cities. N Engl J Med 1993, 329(24):1753-1759.

33. Bennett S: Analysis of survival data by the proportional odds model. Stat Med 1983, 2(2):273-277.

34. Miecznikowski J, Wang D, Liu S, Sucheston L, Gold D: Comparative survival analysis of breast cancer microarray studies identifies important prognostic genetic pathways. BMC Cancer 2010 10(1):573.

35. Horak E, Klenk N, Leek R, LeJeune S, Smith K, Stuart N, Harris A, Greenall M, Stepniewska K: Angiogenesis, assessed by platelet/endothelial cell adhesion molecule antibodies, as indicator of node metastases and survival in breast cancer. Lancet 1992, 340(8828):1120-1124.

36. Guo NL, Wan Y-W, Tosun K, Lin H, Msiska Z, Flynn DC, Remick SC, Vallyathan V, Dowlati A, Shi X, Castranova V, Beer DG, Qian Y: Confirmation of gene expression-based prediction of survival in non-small cell lung cancer. Clin Cancer Res 2008, 14(24):8213-8220.

37. Shedden K, Taylor JM, Enkemann SA, Tsao M-S, Yeatman TJ, Gerald WL, Eschrich S, Jurisica I, Giordano TJ, Misek DE, Chang AC, Zhu CQ, Strumpf D, Hanash S, Shepherd FA, Ding K, Seymour L, Naoki K, Pennell N, Weir B, Verhaak R, Ladd-Acosta C, Golub T, Gruidl M, Sharma A, Szoke J, Zakowski $M$, Rusch V, Kris M, Viale A, et al.: Gene expression-based survival prediction in lung adenocarcinoma: a multi-site, blinded validation study. Nat Med 2008, 14(8):822-827.

38. Wan Y-W, Beer DG, Guo NL: Signaling pathway-based identification of extensive prognostic gene signatures for lung adenocarcinoma. Lung Cancer 2012, 76(1):98-105.

39. Beer DG, Kardia SL, Huang C-C, Giordano TJ, Levin AM, Misek DE, Lin L, Chen G, Gharib TG, Thomas DG, Lizyness ML, Kuick R, Hayasaka S, Taylor $J M$, lannetton MD, Orringer MB, Hanash S: Gene-expression profiles predict survival of patients with lung adenocarcinoma. Nat Med 2002, 8(8):816-824.

40. Comaniciu D, Meer P: Mean shift: a robust approach toward feature space analysis. IEEE Trans Pattern Anal Mach Intell 2002, 24(5):603-619.

41. Cohen LD: On active contour models and balloons. CVGIP: Image Understanding 1991, 53(2):211-218.

42. Haralick RM, Shanmugam K, Dinstein $I H$ : Textural features for image classification. IEEE Trans Syst Man Cybern 1973, SMC-3(6):610-621.

43. Ojala T, Pietikainen M, Maenpaa T: Multiresolution gray-scale and rotation invariant texture classification with local binary patterns. IEEE Trans Pattern Anal Mach Intell 2002, 24(7):971-987.

44. Horng M-H, Sun Y-N, Lin X-Z: Texture feature coding method for classification of liver sonography. Comput Med Imaging Graph 2002, 26(1):33-42.

45. Laws Kl: Rapid texture identification. In Proc. SPIE 0238, Image Processing for Missile Guidance. Volume 238; 1980:376-381. doi:10.1117/12.959169.

46. Leung T, Malik J: Representing and recognizing the visual appearance of materials using three-dimensional textons. Int J Comput Vis 2001, 43(1):29-44.

47. Duan K-B, Rajapakse JC, Wang H, Azuaje F: Multiple svm-rfe for gene selection in cancer classification with expression data. IEEE Trans Nanobioscience 2005, 4(3):228-234.

48. Friedman J, Hastie T, Tibshirani R: Regularization paths for generalized linear models via coordinate descent. J Stat Software 2010, 33(1):1-22

49. Friedman J, Hastie T, Tibshirani R: glmnet: Lasso and elastic-net regularized generalized linear models. R Package Version 2009. [http://cran.r-project.org/web/packages/glmnet/index.html]

50. Breiman L: Random forests. Mach Learn 2001, 45(1):5-32.

51. Liaw A, Wiener M, Breiman L, Cutler A: Package 'randomforest.' Retrieved December 2009, 12:2009.

52. Domingos $P$, Pazzani M: On the optimality of the simple bayesian classifier under zero-one loss. Mach Learn 1997, 29(2-3):103-130.
53. Dimitriadou E, Hornik K, Leisch F, Meyer D, Weingessel A, Leisch MF: The e1071 package. Misc Functions of Department of Statistics (e1071), TU Wien. 2006, [http://cran.r-project.org/web/packages/e1071/index.html]

54. Freund $Y$, Schapire RE: Experiments with a new boosting algorithm. In Machine Learning, Proceedings of the Thirteenth International Conference (ICML). Bary: Morgan Kaufmann; 1996:148-156.

55. Culp M, Johnson K, Michailidis G: ada: An r package for stochastic boosting. J Stat Software 2006, 17(2):9.

56. Yang J, Yu K, Gong Y, Huang T: Linear spatial pyramid matching using sparse coding for image classification. In Computer Vision and Pattern Recognition (CVPR), 2009 IEEE Conference On. Miami, FL: IEEE; 2009:1794-1801.

57. Wang J, Yang J, Yu K, Lv F, Huang T, Gong Y: Locality-constrained linear coding for image classification. In Computer Vision and Pattern Recognition (CVPR), 2010 IEEE Conference On. San Francisco, CA: IEEE; 2010:3360-3367

58. Mensink T, Verbeek J, Perronnin F, Csurka G: Distance-based image classification: Generalizing to new classes at near zero cost. IEEE Trans Pattern Anal Mach Intell 2013, 35(11):2624-2637.

59. Lazebnik S, Schmid C, Ponce J: Beyond bags of features: spatial pyramid matching for recognizing natural scene categories. In Computer Vision and Pattern Recognition (CVPR), 2006 IEEE Conference On, vol. 2. New York; 2006:2169-2178.

60. Tibshirani R: Regression shrinkage and selection via the lasso. J Roy Stat Soc B 1996, 58(1):267-288.

61. Efron B, Hastie $T$, Johnstone I, Tibshirani R: Least angle regression. Ann Stat 2004, 32(2):407-499.

62. Zou H, Hastie T: Regularization and variable selection via the elastic net. J Roy Stat Soc B Stat Meth 2005, 67(2):301-320.

63. Bühlmann $P, Y u$ B: Boosting with the I 2 loss: regression and classification. J Am Stat Assoc 2003, 98(462):324-339.

64. Hoerl $A E$, Kennard RW: Ridge regression: biased estimation for nonorthogonal problems. Technometrics 1970, 12(1):55-67.

65. Binder $\mathrm{H}$, Schumacher $\mathrm{M}$ : Allowing for mandatory covariates in boosting estimation of sparse high-dimensional survival models. BMC Bioinformatics 2008, 9:14.

66. Tutz $\mathrm{G}$, Binder $\mathrm{H}$ : Boosting ridge regression. Comput Stat Data Anal 2007, 51(12):6044-6059

67. Binder $\mathrm{H}$, Schumacher $\mathrm{M}$ : Incorporating pathway information into boosting estimation of high-dimensional risk prediction models. BMC Bioinformatics 2009, 10:18.

doi:10.1186/1471-2105-15-310

Cite this article as: Wang et al:: Novel image markers for non-small cell lung cancer classification and survival prediction. BMC Bioinformatics 2014 15:310

\section{Submit your next manuscript to BioMed Central} and take full advantage of:

- Convenient online submission

- Thorough peer review

- No space constraints or color figure charges

- Immediate publication on acceptance

- Inclusion in PubMed, CAS, Scopus and Google Scholar

- Research which is freely available for redistribution
Ciomed Central 